\title{
Evaluation of prescription pattern of psycholeptic medications at hospital discharge for patients with cardiovascular diseases in national cardiovascular disease hospital of Bangladesh
}

\author{
Mir Muhammad Nasir Uddin ${ }^{1 *}$, Mohammad Shah Hafez Kabir ${ }^{2}$, Md. Mamun Al-Amin ${ }^{3,4}$, Mynul Hasan ${ }^{3}$, \\ Talha Bin Emran ${ }^{5}$, Mohammad Touhidul Islam ${ }^{6}$, Md Ashiqul Islam $^{1}$, Shahriar Ahmed ${ }^{1}$ \\ ${ }^{1}$ Department of Pharmacy, University of Chittagong, Chittagong 4331, Bangladesh. \\ ${ }^{2}$ Department of Pharmacy, International Islamic University Chittagong, Chittagong-4203, Bangladesh. \\ ${ }^{3}$ Department of Pharmaceutical Sciences, North South University, Bashundhara, Dhaka 1229, Bangladesh. \\ ${ }^{4}$ Queensland Brain Institute, University of Queensland, Australia. \\ ${ }_{5}^{5}$ Laboratory of Vaccinology and Applied Immunology, Kanazawa University School of Pharmacy, Kakuma-machi, Kanazawa 920-1192, Japan. \\ ${ }^{6}$ Department of Pharmaceutical Technology, University of Dhaka, Dhaka, Bangladesh.
}

\section{ARTICLE INFO}

Article history:

Received on: 23/09/2016

Accepted on: 11/11/2016

Available online: 30/03/2017

\section{Key words:}

Psycholeptic;

benzodiazepine;

bromazepam, polypharmacy.

\begin{abstract}
Background: The association of mental disorder with cardiac disease is not a coincidence rather a strong relationship exists in between those two.

Objective: We investigated the prescription pattern of psycholeptic drugs during the discharge of patients in a national cardiovascular hospital.

Methods: Data was collected from the National Institute of Cardiovascular Diseases (NICVD) at Dhaka in Bangladesh. In total, 1076 prescriptions were collected randomly from July 2012 to June 2013. Data were analyzed by using the software Statistical Package for Social Science (SPSS, version 22).

Results: Benzodiazepine was the highest $(99.3 \%)$ prescribed psycholeptic class while Bromazepam $(87.49 \%)$ the highest advised molecule. Psycholeptics were advised for 15 (38.77\%), $10(19.91 \%), 30(15.72 \%)$ and 7 $(13.39 \%)$ days. The patient group without psycholeptic medication $(\mathrm{M}=6.64$ day) spent significantly $(\mathrm{p}<0.05)$ a longer duration in the hospital compared to the psycholeptic medication (Mean=5.06 day) group. Age is significantly $(\mathrm{p}<0.05)$ associated with the duration of staying in hospital and polymorbidity. Also, polymorbidity $(p<0.05)$ is significantly associated with the polypharmacy. No significant $(p<0.05)$ correlation has been found between age and polypharmacy. Duration of psycholeptics intake was not significantly $(p<0.05)$ related to either age, or polymorbidity,or length hospital stay. Conclusion: This study revealed that bromazepam is the utmost prescribed drug of choice at NICVD, Bangladesh. Frequent prescription of bromazepam indicates that this kind of patients' needs enough sleep to reduce their mental stress.
\end{abstract}

\section{INTRODUCTION}

Cardiovascular disease (CVD) costs nearly 17.3 million people around the world every year (WHO, 2011). Among them, $80 \%$ are from low and middle-income nations. The death toll might be increased up to 23.3 million by 2030 (Mathers and

\section{* Corresponding Author}

Mir Muhammad Nasir Uddin, Assistant Professor, Department of

Pharmacy, University of Chittagong, Chittagong-4331, Bangladesh

Email: mir.m.nasiruddin @ gmail.com; Phone: 008801712725987
Loncar, 2006). Major risk factors that may aggravate cardiovascular disease are obesity, hypertension, high cholesterol and high glucose. Additional factors including mental illness also contribute to the cardiovascular disease. Accumulating evidence suggests that depression and anxiety level are closely linked to heart disease (Chen et al., 2013; Elderon and Whooley, 2013; Nakamura et al., 2013; Thurston et al., 2013). Extremely stressful events radically raise cardiac morbidity as well as cardiac mortality. Study surveyed on adults who are residing in 17 countries in the world including Europe, Asia, and Americas. 
The authors reported an association between anxiety and mood disorders among the patient with cardiovascular disease (Ormel et al., 2007). Chronic stress including the mental stress from workplace enhances the risk of coronary heart disease (CHD). Hypertension is the commonest phenomenon observed due to the extreme stress and anxiety in daily life (Hildrum et al., 2008; Player and Peterson, 2011). There exists a huge published reports to the correlation of stress with coronary artery disease (CAD) (Bunevicius et al., 2013; Roest et al., 2010), implanted cardioverter-defibrillators (ICD) (Hegel et al., 1997; Luyster et al., 2006; Sola and Bostwick, 2005) and congestive heart failure (CHF) (Kulcu et al., 2007; Serafini et al., 2010).

Sleep disorder and CVD both affect each other bidirectionally. Sleep deprivation is strongly associated with CVD since it causes hypertension. On the contrary, CVD disrupts normal sleep; e.g., patients with CHF suffers paroxysmal nocturnal dyspnea (PND), a state where shortens of breath usually occurs at night. Thus patients become awake and cannot sleep. Sleep apnea is associated with hypertension and diabetes, putting these patients at high risk of developing CVD(Herrscher et al., 2013). Moreover, sufficient sleep duration is independently connected with a lower risk of composite and fatal CVD(Hoevenaar-Blom et al., 2013). Additionally, short sleep duration increases many major cardiac risk factors such as overweight, obesity, hypertension, total cholesterol and triglycerides(Ikehara et al., 2009).

A large variety of generics is necessary for the management of CVD. According to the Anatomical Therapeutic Chemical (ATC) Classification System these therapeutic classes are antihypertensives, (C02), diuretics (C03), peripheral vasodilators ( $\mathrm{C} 04)$, vasoprotectives (C05), beta blocking agents (C07), calcium channel blockers (C08), agents acting on the reninangiotensin system, (C09) lipid modifying agents (C10)(Al-Amin et al., 2012). Besides these drugs for acid-related disorders (A02), psycholeptics (N05), vitamins (A11) and mineral supplements (A12) are prescribed for the treatment of CVD. Among the psycholeptics, anxiolytics (N05B) such as Benzodiazepine derivatives (N05BA) are advised in most of the instances. However, the prescription pattern of psycholeptic drugs at hospital discharge, especially in Bangladesh, has not yet been documented.

This study identified the major psycholeptic class prescribed for the management of cardiovascular disease; (ii) identified the major psycholeptic drugs prescribed for the management of heart disease; (iii) analyzed the presence of psycholeptic medications in prescription; (iv) assessed the association and psycholeptics intake with age, polypharmacy, polymorbidity, duration of hospital stay; (v) analyzed the correlation of psycholeptics intake duration with age, polypharmacy, polymorbidity, length of hospital stay; (vi) discussed the necessity of psycholeptics in the treatment of cardiovascular diseases notably hypertension (HT), acute myocardial infarction (AMI), angina pectoris (AP), atrial septal defect (ASD), coronary artery bypass graft (CABG), ischemic cardiomyopathy (IC), ischemic heart disease (IHD), left ventricular dysfunction (LVD), left ventricular failure(LVF), myocardial refraction (MR),mitral stenosis (MS), non-ST segment myocardial infarction (NSTSMI), pulmonary hypertension $(\mathrm{PH})$, pulmonic stenosis (PS), stable angina (SA), sinus bradycardia (SB) and unstable angina (UA).

\section{MATERIALS AND METHODS}

\section{Study design}

The cross-sectional study was conducted at the National Institute of Cardiovascular Diseases (NICVD) in Bangladesh. NICVD is a specialized hospital for cardiovascular disease management. It is the most renowned health care institution containing 431-beds located at Sher-e-Bangla Nagar in the capital of Bangladesh (Banglapedia, 2012). Patients choose to get admission at this hospital due to the high-quality health care at low cost.

A report demonstrated that around 900 indoor patients are admitted at NICVD while nearly 800 - 900 outdoor patients received treatment every day from the emergency and outside service. Moreover, approximately 150 patients are being admitted and released per day (Uzzal, 2014).

\section{Sampling technique}

Data were collected from the patients $(n=1076)$ who were admitted during the period from $1^{\text {st }}$ July 2012 to $30^{\text {th }}$ June 2013 at the National Institute of Cardiovascular Diseases (NICVD) in Bangladesh. NICVD hospital has 431 beds where each patient requires on an average a length of five (05) days for getting the treatment (Majumder, 2014). So, this hospital provides health service to the 31463 in-bed patients per year. Hence, this study aims to describe a population of cardiovascular prescription $(n=1076)$ within the population $(n=31463)$ by surveyed prescription.

The prescription has covered the total of around $3.42 \%$ population. This sample was taken by $95 \%$ confidence level and 1.06 confidence intervals.

\section{Variables}

Demographic information of every patient: age, gender, hospital admission, discharge dates, drugs prescribed, disease identified were recorded. Disease types were assessed in fulfillment with the $10^{\text {th }}$ Revision of the International Classification of Diseases (ICD-10) (WHO, 2012b). The patient who have four or more diseases considered as polymorbid (Wawruch et al., 2008) and who were advised five or more drugs found as polypharmacy. We have followed the Anatomical Therapeutic Chemical classification (ATC) for determining drug classes (WHO, 2012a).

The number of medications prescribed by the physician was recorded and compared for each of the patients. There were many mixtures of active pharmaceutical ingredient (API), present in the prescription. Patient was grouped into 10-year age brackets (Below 20 years; 21-30; 31-40; 41-50; 51-60; 61-70; 71-80; Above 80 years). 


\section{Participant selection procedure, inclusion criteria}

We went to visit National Institute of Cardiovascular Diseases (NICVD) in Bangladesh. Our selection principles were (i) should be a patient with the cardiovascular disorder, (ii) should have minimum one cardiovascular disease, (iii) discharged from the NICVD hospital, (iv) participants who was admitted to this hospital in the bed.

\section{Consent form and Ethical Issues}

The aim, objectives, and procedures of the present study were described to the participants before collection of data. The participants were approved verbally to copy their prescription from the nearest Xerox shop. The institutional review board of North South University approved this cross-sectional study (Approval Number: 2013/NSU/HR/00123/MN334).

\section{Statistical analysis}

All the data presented as mean \pm standard deviation (SD) value. Categorical variables were expressed by frequencies and percentages. All statistical tests were performed at a significance level lower than 0.05 in SPSS (version 22, IBM Software Inc, USA) and GRAPHPAD PRISM® (version 6.02; GraphPad Software Inc., San Diego, CA, USA). Moreover, we performed linear regression analysis between age and polypharmacy; age and polymorbidity; age and length of hospital stay, etc.

\section{RESULTS}

The analysis of our data showed that male (78\%)and female $(22 \%)$ of mean age $53.87 \pm 13.12$ (mean \pm SD)were discharged from the hospital. A minimum of 3 years and maximum 96 years old patient was discharged. Moreover, seven patients were below 20years old while 11 patients were greater than 80 years old. The average age of male $(54.05 \pm 12.84)$ and female (53.21 \pm 14.09$)$ CVD patients was almost equal.

Patients suffer a minimum number of one to maximum six diseases; mean disease was $2.49 \pm 1.1$ (mean \pm SD). The average number of drugs advised by the physicians was $8.56 \pm 1.93$ (mean \pm $\mathrm{SD})$.We found a minimum number of 3 drugs that were prescribed in 1 prescription while a maximum of 14 medications was prescribed in 5 prescriptions. No gender difference was found in some drugs advised between the male (8.52) and female (8.49) patients. The patient had spent a minimum of 1 to a maximum of 50 days in the hospital; average duration in the hospital 5.35 \pm 5.1 (mean $\pm \mathrm{SD})$.

Blood pressure data showed no difference between psycholeptic and non-psycholeptic groups. In psycholeptic group, mean systolic blood pressure was $120.5 \pm 10.5$ while mean diastolic pressure was $82 \pm 5.6(\mathrm{~mm} / \mathrm{Hg})$. In the case of a non-psycholeptic group, mean systolic blood pressure was $120.5 \pm 10.7$ while diastolic pressure was $83 \pm 5.9(\mathrm{~mm} / \mathrm{Hg})$. Mean arterial pressure (MAP) did not significantly differ in between psycholeptic $(94.66 \pm 7.23)$ and non-psycholeptic groups $(96.16 \pm 11.63)$. Pulse rate was higher than healthy people (72) in both psycholeptic
$(83 \pm 11.2)$ and non-psycholeptic $(84 \pm 12.1)$ groups. There were no significant differences observed for HDL, LDL, Total Cholesterol and Triglyceride level in the plasma between psycholeptic and non-psycholeptic groups.

We noted that the medication prescribed to discharge patients are anti-coagulant combination (e.g., clopidogrel+aspirin), lipid modifying agents(e.g., atorvastatin), anti-hypertensive (e.g., ramipril), anti-anginal (e.g., nitroglycerin), beta-blockers (e.g., carvedilol), other cardiac preparation (e.g., trimetazidine), diuretic combination (e.g., hydrochlorothiazide+furosemide), Drugs for acid-related disorders (e.g., omeprazole), benzodiazepine (e.g., bromazepam). The analysis of CVD patients with or without psycholeptics is summarized in Table 1 .

Table 1: Characteristics of CVD patients with and without psycholeptics $(\mathrm{n}=1083)$

\begin{tabular}{|c|c|c|}
\hline Characteristics & $\begin{array}{c}\text { CVD patients with } \\
\text { Psycholeptics }\end{array}$ & $\begin{array}{c}\text { CVD patients } \\
\text { without } \\
\text { Psycholeptics }\end{array}$ \\
\hline Patients & 887 & 196 \\
\hline Male & 715 & 130 \\
\hline Female & 171 & 66 \\
\hline Smoking ( $\%$ of patient) & $73 \%$ & $72 \%$ \\
\hline \multicolumn{3}{|l|}{ Blood Pressure $(\mathrm{mm} / \mathrm{Hg})$} \\
\hline Systolic & $120.5 \pm 10.5$ & $120.5 \pm 10.7$ \\
\hline Diastolic & $82 \pm 5.6$ & $83 \pm 5.9$ \\
\hline Pulse & $83 \pm 11.2$ & $84 \pm 12.1$ \\
\hline \multicolumn{3}{|l|}{ Lipid profile } \\
\hline HDL (mg/dl) & $38.4 \pm 4.0$ & $38.4 \pm 4.0$ \\
\hline $\mathrm{LDL}(\mathrm{mg} / \mathrm{dl})$ & $127.8 \pm 17.0$ & $126.8 \pm 17.0$ \\
\hline Triglyceride (mg/dl) & $232.8 \pm 68.0$ & $223.4 \pm 56.0$ \\
\hline Total Cholesterol (mg/dl) & $216.5 \pm 52.0$ & $213.5 \pm 62.0$ \\
\hline RBS & $10.95 \pm 7.59$ & $10.42 \pm 7.05$ \\
\hline Creatinine & $1.24 \pm 0.38$ & $1.23 \pm 0.05$ \\
\hline Age (years) [Mean (SD)] & $53.8 \pm 12.3(10-95)$ & $54.1 \pm 16.5(3-96)$ \\
\hline$<20$ & 2 & 8 \\
\hline $21-30$ & 22 & 11 \\
\hline $31-40$ & 96 & 16 \\
\hline $41-50$ & 289 & 42 \\
\hline $51-60$ & 275 & 54 \\
\hline $61-70$ & 131 & 43 \\
\hline $71-80$ & 57 & 16 \\
\hline$>80$ & 15 & 6 \\
\hline Polypharmacy & $95.71 \%(\mathrm{n}=849)$ & $89.79 \%(n=176)$ \\
\hline Non Polypharmacy & $4.92 \%(\mathrm{n}=38)$ & $10.21 \%(n=20)$ \\
\hline
\end{tabular}

Polymorbidity and polypharmacy were not significantly affecting in CVD patients with or without psycholeptics (fig. 1). Interestingly, the length of hospital stay was significantly $(\mathrm{p}<0.05)$ lower in CVD patients with psycholeptics prescription than without psycholeptics.

Psycholeptics was given to the 887 patients $(81.91 \%)$ out of 1083 patients. Most of the prescribed psycholeptics (98.09\%) are under benzodiazepines groups (Table 2). The other suggested drugs $(1.91 \%)$ belong to antidepressants, anti-vertigo, antiparkinson, nootropic and anti-epileptics. We have analyzed the ratio between the number of prescription and number of psycholeptics prescribed. The ratio between total drugs and psycholeptic drugs on an average in each prescription was 8.56:0.82. 
Table 2: Types of psycholeptics prescribed for CVD patient ( $\mathrm{n}=887)$.

\begin{tabular}{llr} 
Therapeutic Class & \multicolumn{1}{c}{ Generic } & $\begin{array}{c}\text { Number of patients } \\
\text { with prescribed } \\
\text { drugs }(\%)\end{array}$ \\
\hline & Bromazepam & $776(87.49)$ \\
Benzodiazepine & Clonazepam & $61(6.88)$ \\
& Midazolam & $23(2.59)$ \\
& Alprazolam & $10(1.13)$ \\
\hline Antipsychotic+ & Flupenthixol+ Melitracen & $6(0.68)$ \\
Antidepressant & Escitalopram & $1(0.11)$ \\
\hline \multirow{2}{*}{ Antivertigo } & Flunarizine & $3(0.34)$ \\
& Cinnarizine & $2(0.23)$ \\
\hline Anti-Parkinson & Procyclidine & $3(0.34)$ \\
\hline Nootropic & Piracetam & $1(0.11)$ \\
\hline Antiepileptic & Carbamazepine & $1(0.11)$ \\
\hline
\end{tabular}

Bromazepam was the most $(87.49 \%)$ advised generic for the CVD patient followed by Clonazepam (6.88\%), midazolam $(2.59 \%)$, alprazolam $(1.13 \%)$ and flupenthixol plus melitracen combination $(0.68 \%)$. Flunarizine and procyclidine were prescribed for 3 CVD patients. Cinnarizine was suggested for two patients. Escitalopram, piracetam, and carbamazepine were found only one occasion. Most of the physicians recommended benzodiazepines for 15 days (38.77\%) followed by $10(19.91 \%)$, 30 (15.72\%), 7 (13.39\%) days (figure 1) and 60 days $(4.31 \%)$ (Table 3). Interestingly, "NM" (Nothing Mentioned) (3.38\%) and "continue" $(1.98 \%)$ were also found. Benzodiazepines were suggested as solid dosage forms (tablet) and should be taken before sleep at night. We analyzed the position of psycholeptics in the prescription. Except few cases $(3.5 \%)$, most benzodiazepines $(96.5 \%)$ were written as the last medication on the prescription. Analysis of benzodiazepines in various age brackets showed that they were mostly advised in 41-50 and 51-60 age groups (fig. 2). There were very limited instances where these medications were prescribed for below 20 years and over 80 years age bracket. Benzodiazepines were advised to the 31-40, 61-70 and 71-80 age groups in similar fashions.

From linear regression analysis we have found that the prescription data showed the great length of hospital stay in response to age $\left(\mathrm{R}^{2}=0.004, \mathrm{p}<0.05\right)$ (Fig 3). As age is increased patient, need more nursing time from the hospital. Also, there was a significant relation between the age and disease $\left(\mathrm{R}^{2}=0.006\right.$, $\mathrm{p}<0.05)$. As age is increased patient suffer more disease (polymorbid condition). Also, some diseases are related to the number of drugs $\left(\mathrm{R}^{2}=0.007, \mathrm{p}<0.05\right)$. Polymorbidity is associated with polypharmacy. There was no correlation between age vs a number of drug intake; age vs duration of psycholeptics intake; the number of disease vs length of psycholeptics intake; the number of disease vs length of hospital stay; the number of drug intake vs length of hospital stay; length of psycholeptics intakes length of hospital stay.

Table 3: Duration of Benzodiazepines advised for the CVD patients ( $\mathrm{n}=859)$.

\begin{tabular}{|c|c|c|c|c|c|}
\hline \multirow[t]{2}{*}{ Duration (day) } & Bromazepam & Clonazepam & Midazolam & Alprazolam & Benzodiazepines \\
\hline & No. of drugs (\%) & No. of drugs (\%) & No. of drugs (\%) & No. of drugs (\%) & No. of drugs (\%) \\
\hline 5 & $1(0.1)$ & $1(1.6)$ & - & - & $2(0.23)$ \\
\hline 7 & $104(13.4)$ & $8(13.1)$ & $3(13.0)$ & - & 115 (13.39) \\
\hline 10 & $163(21.0)$ & $1(1.6)$ & $6(26.1)$ & $1(10.0)$ & $171(19.91)$ \\
\hline 14 & $3(0.4)$ & - & $3(13.0)$ & - & $6(0.70)$ \\
\hline 15 & $322(41.5)$ & $10(16.4)$ & $8(34.8)$ & $1(10.0)$ & $333(38.77)$ \\
\hline 20 & $12(1.5)$ & - & $1(4.3)$ & - & $13(1.51)$ \\
\hline 21 & - & $1(1.6)$ & - & - & $1(0.12)$ \\
\hline 30 & $127(16.4)$ & $1(1.6)$ & $1(4.3)$ & $6(60.0)$ & $135(15.72)$ \\
\hline 60 & $9(1.2)$ & $26(42.6)$ & - & $2(20.0)$ & $37(4.31)$ \\
\hline $\mathrm{NM}^{*}$ & $18(2.3)$ & $10(16.4)$ & $1(4.3)$ & - & $29(3.38)$ \\
\hline $\mathrm{C}^{* *}$ & $17(2.2)$ & - & - & - & $17(1.98)$ \\
\hline
\end{tabular}

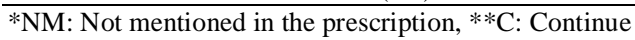

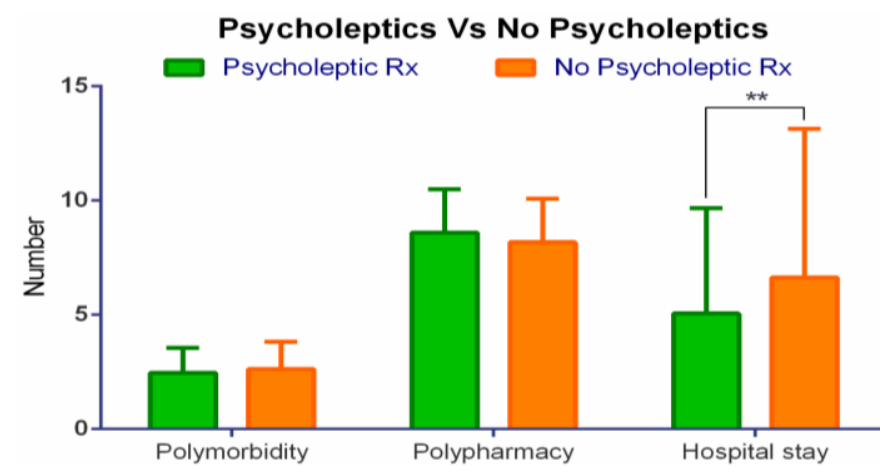

Fig. 1: Psycholeptics in various age brackets. The horizontal axis represents age groups in 10 years age brackets. The vertical axis represents the frequency of duration in $5,7,10,14,15,20,21,30,60,90$ days, not mentioned (111) and to continue (365).

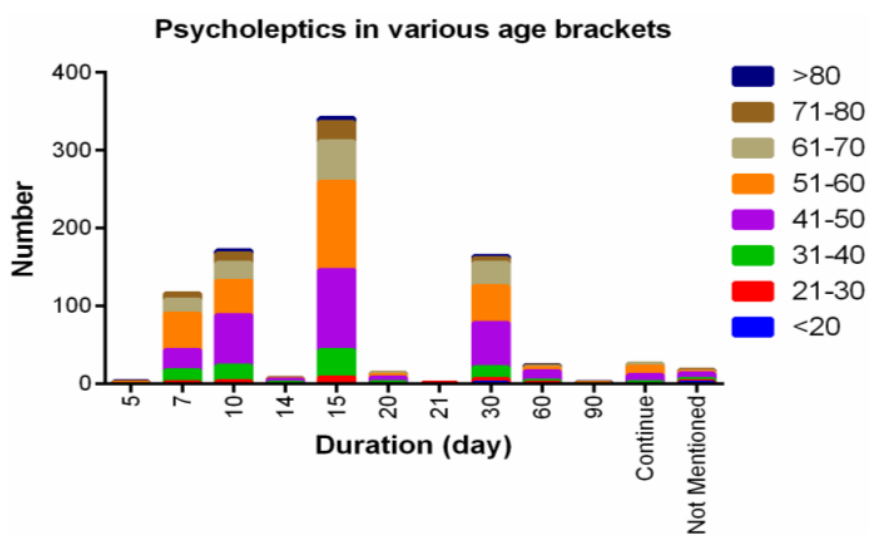

Fig. 2: Psycholeptics in various age brackets. The horizontal axis represents age groups in 10 years age brackets. 

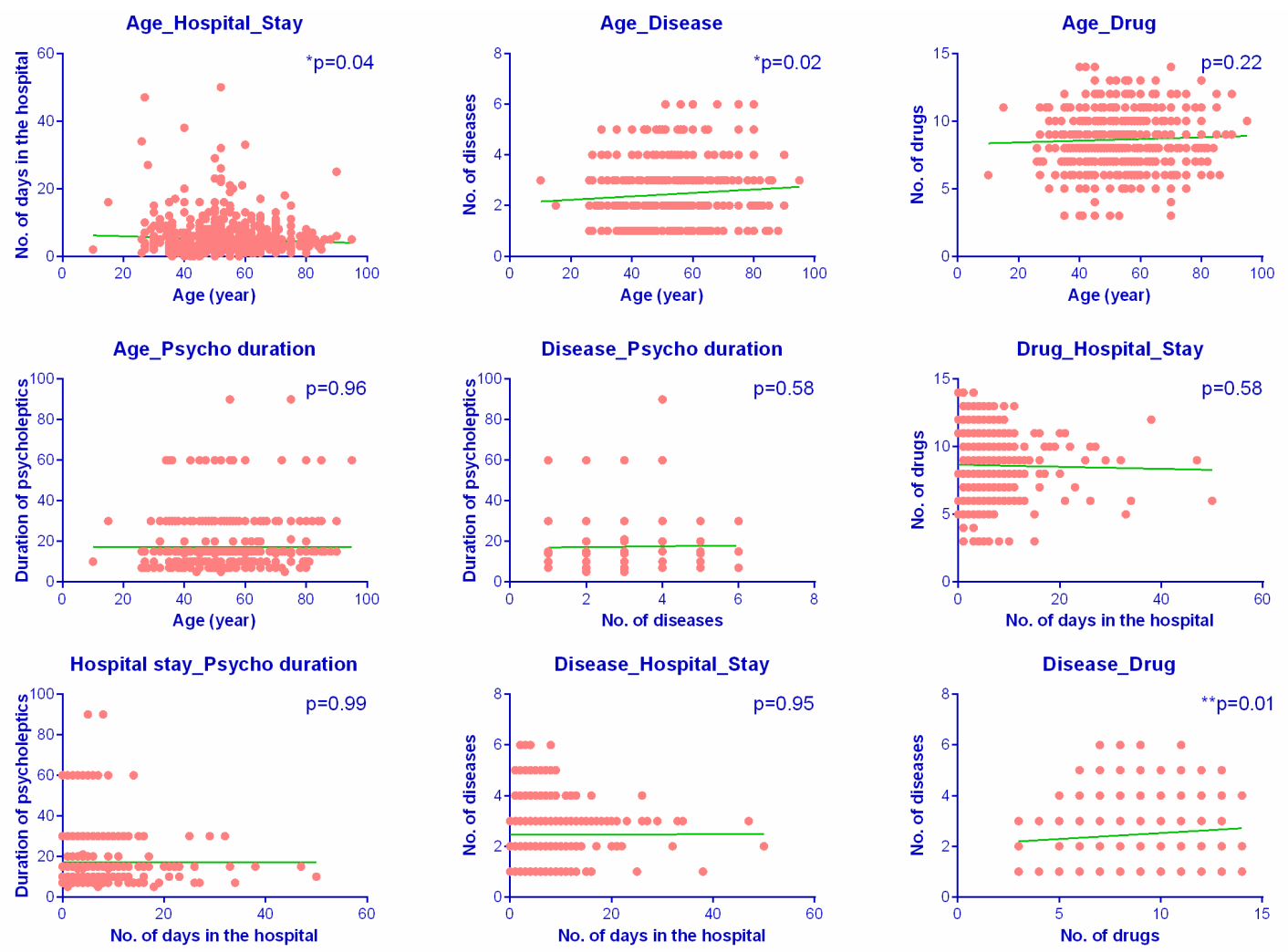

Fig. 3: Psycholeptics in various age brackets. The horizontal axis represents age groups in 10 years age brackets.

\section{DISCUSSION}

The result of the present study showed that a significant number of generics were advised to the patient with cardiovascular disease. In most of the cases, more than eight drugs were suggested. Highest prescribed psycholeptic class and molecules were Benzodiazepine and Bromazepam. Psycholeptics were advised for the duration of 15, 10, 30 and seven days. Patient without psycholeptic medication had an extended length of hospital stay than the psycholeptic medication. Age was associated with the duration of hospital stay and polymorbidity. Also, polymorbidity is associated with the polypharmacy. There was no association between age and polypharmacy. Duration of psycholeptics intake was not related to neither age nor polymorbidity nor length of hospital stay.

The treatment of cardiovascular disease may depend on multiple factors. Prescribing one molecule is not enough for the management and treatment of this kind of chronic illness. Various factors should consider while writing the prescriptions; for example, high blood pressure, high cholesterol level, water volume, coagulating factors, nutrition, mental health, and rest.

Our present study demonstrated that most of the patients are advised to take one psycholeptic, and that is a benzodiazepine (e.g., bromazepam). The age range of prescribing benzodiazepine was 31 to 80 years. There were several instances where benzodiazepines were prescribed to below 30 and over 80 years. Probably benzodiazepine offers very limited therapeutic effect and more prone to show side effects in this stage of life
(Windle et al., 2007). Additionally, these people take other cardiac medications. So, advising a large number of drugs will create a perilous situation where patients would be more susceptible to drug interaction, polypharmacy and side effects. The analysis of prescription data showed that blood pressure, lipid profile, blood glucose levels (RBS) and serum creatinine was not varied in between psycholeptic medication presence or absence in the prescription. Lipid profiles of both psycholeptic and nonpsycholeptic medication containing prescriptions were in the same level. The present finding is consistent with the previous report (Khan and Majumder, 2011). We observed a high pulse rate in both groups of cardiovascular patients than healthy people. The result of the current study demonstrated that CVD patients with psycholeptics had spent shorter length in the hospital. This finding can be interpreted as; patients spending long duration in the hospital get received more nursing care and more time to restore their normal body function. We found an association of age with polymorbidity, age, and length of hospital stay, polymorbidity and polypharmacy. Interestingly, the length of suggested psychiatric medication treatment was not associated with either age nor polymorbidity or length in the hospital stay. Our result showed that middle-aged people are mostly suffering cardiovascular disease. It may happen since CVD risk factors such as smoking, sedentary lifestyle, cholesterol, obesity, uncontrolled food habits gradually facilitates to develop cardiac disease. These people are not being noticed until they have the first incident like first heart attack, hypertension, stroke, angina pain, etc. We found that males are more prone to cardiac disease than female. This may be 
explained by the association of cardiac risk factors including smoking and male hormones. In developed and developing countries male was reported to smoke more than their female counterpart (Mackay and Amos, 2003). The most important point to emerge from our present study is the common prescribing habit of benzodiazepines. Bromazepam was the mostly advised generic for the patient followed by clonazepam, midazolam, and alprazolam. The appearance of benzodiazepines in almost every prescription was observed in the age range between 31 to 70 years indicating the requirement of sleep and rest for discharged patients with cardiovascular diseases. A review study assesses the relationship between duration of sleep and morbidity and mortality from coronary heart disease (CHD), stroke, and total cardiovascular disease. The study revealed that the duration of sleep is predictors, or markers, of cardiovascular outcomes(Cappuccio et al., 2011b). In another study, the role of benzodiazepines in $\mathrm{CHD}$ and other CVD were investigated (Williams Jr, 1990). The authors suggest that benzodiazepines might control angina and silent ischemia and another cardiac disease by blunting the responses arise from stress hormones. Benzodiazepine (alprazolam) reduces the duration of silent ischemia by $70 \%$ in coronary patients(Shell and Swan, 1986). The therapeutic benefit of alprazolam was explained as they help to reduce stress induced by adrenaline, noradrenalin, and corticosteroids. Benzodiazepine has been prescribed for the postmyocardial infarction for a long time(Strik et al., 2001). Most of the studies conclude that benzodiazepines are beneficial since they reduce the level of anxiety, stress hormone response and help people sleep for a long duration (Vogel et al., 1984; Williams, 1990). Insufficient sleep is an independent risk factor for CVD (Cappuccio et al., 2011a; Hoevenaar-Blom et al., 2011). Reduced sleep duration is associated with composite and fatal CVD since they cause higher risk of obesity, overweight, high total cholesterol, high HbA1c (glycosylated hemoglobin), triglycerides and high blood pressure. Also, inadequate sleep duration causes sustained low-grade inflammation and increased leptin level in the blood which accelerates appetite and little energy expenditure. These two factors (higher appetite and less energy expenditure) facilitate to develop obesity and higher cholesterol and glycemic level. Importantly, sufficient sleep reduces cardiovascular morbidity and mortality (Hoevenaar-Blom et al., 2013; Ikehara et al., 2009).

Recently a "Morgen study" was conducted in the Netherlands by Hoevenaar-Blom and co-workers. The authors reported that insufficient sleep is associated with the low level of inflammation in turn appetite enhance and energy expenditure reduces leads to higher glycemic level and obesity.Interestingly, another study report that both short and long duration of sleep is associated with the cardiac mortality (Ikehara et al., 2009). The advising of at least one psycholeptic drugs is observed as a common habit of a cardiologist. In this circumstance, cardiologist should consider several limitations of psycholeptics in CVD patients. Psychotropic drug has lethal cardiac side effects (Longo and Johnson, 2000). Benzodiazepines showed a variety of side effects including slowed psychomotor performance, ataxia, hip fracture and daytime sedation (Gleason et al., 1998). A cardiologist should remember some of the important cardiac factors in the safer selection of psychotropic drugs for the vulnerable patient in their daily clinical practice (O'Brien and Oyebode, 2003). Finally, a cardiologist should remember both positive and negative therapeutic benefits of psychotropic medicines in the cardiovascular system and follow the standard guidance for the practical management of psychotropic symptom in CVD patients (Mackin, 2008). To our knowledge, the findings of our present study are novel in many respects. Firstly; we report the prescribing pattern of psycholeptics to the discharged CVD patients in the hospital. The adherence and the necessity of benzodiazepines are discussed. Secondly; we have analyzed the duration of psycholeptics for the management of cardiovascular disease. Thirdly; middle age group is the primary target patient where cardiologist suggests benzodiazepine. There were several limitations of the present study. We analyzed around 1000 patients and included only one hospital. However, this hospital is the most modern cardiovascular hospital in Bangladesh. But we thought that, surveying only NICVD hospital is enough to investigate our current hypothesis. Around 800-900 CVD outdoor patients come in every day to this hospital from the outside of the Dhaka and rural areas.

There are limited resources and facilities to manage cardiovascular disease at the hospital that is located outside of the Dhaka. Physicians recommend their CVD patients to the NICVD. Moreover, managing CVD disease is costly except treating at NICVD. Due to the limited statistical knowledge, we conducted several tests on our data. Since we have collected data from the prescription of discharged patient, therefore we failed to measure sleep duration of the patient. Monitoring of sleep duration could be investigated in the future study. Also, prospective study will include big sample size with more variables from a large number of hospitals. Finally, we were not able to follow up the patient's biochemical parameters such as lipid profile, blood pressure. This was important to measure the outcome of psycholeptics in the patient with a cardiovascular disorder.

\section{CONCLUSION}

The aim of the present study was to investigate the prescribing pattern of the psycholeptics by the cardiologist in the hospital discharged patients with cardiovascular disease. Among the psycholeptics bromazepam was the most frequent prescribing drug molecule. The literature review showed that the addition of benzodiazepine with the cardiovascular medications could bring beneficial effect by providing sufficient sleep which in turn reduces anxiety, stress hormone response, and stress induced lowgrade inflammation.

\section{Financial support and sponsorship: Nil.}

Conflict of Interests: There are no conflicts of interest. 


\section{REFERENCES}

Al-Amin MM, Zinchenko A, Rana MS, Uddin MMN, IMSP. Study on Polypharmacy in Patients with Cardiovascular Diseases. Journal of Applied Pharmaceutical Science, 2012; 2:53-60.

Banglapedia. 2012. National Institute of Cardiovascular Diseases. Banglapedia (National Encyclopedia of Bangladesh), Dhaka.

Bunevicius A, Staniute M, Brozaitiene J, Pop VJ, Neverauskas J, Bunevicius R. Screening for anxiety disorders in patients with coronary artery disease. Health Qual Life Outcomes, 2013; 11:37.

Cappuccio FP, Cooper D, D'Elia L, Strazzullo P, Miller MA. Sleep duration predicts cardiovascular outcomes: a systematic review and meta-analysis of prospective studies. Eur Heart J, 2011; 32:1484-1492.

Cappuccio FP, Cooper D, D'Elia L, Strazzullo P, Miller MA. Sleep duration predicts cardiovascular outcomes: a systematic review and meta-analysis of prospective studies. European Heart Journal, 2011.

Chen H, Zhang L, Zhang M, Song X, Zhang H, Liu Y, Lv S. Relationship of depression, stress and endothelial function in stable angina patients. Physiol Behav, 2013; 118:152-158.

Elderon L, Whooley MA. Depression and cardiovascular disease. Prog Cardiovasc Dis, 2013; 55:511-523.

Gleason P, Schulz R, Smith N, Newsom J, Kroboth P, Kroboth F, Psaty B. Correlates and prevalence of benzodiazepine use in community-dwelling elderly. J Gen Intern Med, 1998; 13:243-250.

Hegel MT, Griegel LE, Black C, Goulden L, Ozahowski T. Anxiety and depression in patients receiving implanted cardioverterdefibrillators: a longitudinal investigation. Int J Psychiatry Med, 1997; 27:57-69.

Herrscher TE, Overland B, Sandvik L, Westheim AS, Akre H. High cardiovascular risk profile in patients with sleep apnea. Laryngoscope, 2013.

Hildrum B, Mykletun A, Holmen J, Dahl AA. Effect of anxiety and depression on blood pressure: 11-year longitudinal population study. The British journal of psychiatry : the journal of mental science, 2008; 193:108-113.

Hoevenaar-Blom MP, Spijkerman AM, Kromhout D, van den Berg JF, Verschuren WM. Sleep duration and sleep quality in relation to 12-year cardiovascular disease incidence: the MORGEN study. Sleep, 2011; 34:1487-1492.

Hoevenaar-Blom MP, Spijkerman M, Kromhout D, Verschuren WM. Sufficient sleep duration contributes to lower cardiovascular disease risk in addition to four traditional lifestyle factors: the MORGEN study. Eur J Prev Cardiol, 2013.

Ikehara S, Iso H, Date C, Kikuchi S, Watanabe S, Wada S, Inaba Y, Tamakoshi A. Association of sleep duration with mortality from cardiovascular disease and other causes for Japanese men and women: the JACC study. Sleep, 2009; 32:295-301.

Khan A, Majumder A. Study of Lipid Profile and Coronary Angiographic Pattern in Young Bangladeshi Patients with Acute Coronary Syndrome, 2011.

Kulcu DG, Kurtais Y, Tur BS, Gulec S, Seckin B. The effect of cardiac rehabilitation on quality of life, anxiety and depression in patients with congestive heart failure. A randomized controlled trial, short-term results. Eura Medicophys, 2007; 43:489-497.

Longo LP, Johnson B. Addiction: Part I. Benzodiazepines--side effects, abuse risk and alternatives. Am Fam Physician, 2000; 61:21212128 .

Luyster FS, Hughes JW, Waechter D, Josephson R. Resource loss predicts depression and anxiety among patients treated with an implantable cardioverter defibrillator. Psychosomatic medicine, 2006; 68:794-800. 8:123-130

Mackay J, Amos A. Women and tobacco. Respirology, 2003;

Mackin P. Cardiac side effects of psychiatric drugs. Hum Psychopharmacol, 2008; 1:3-14

Majumder AAS. 2014. Health Bulletin 2014. Ministry of Health and Family Welfare (MOHFW), Sher-e-Bangla Nagar- Dhaka.

Mathers CD, Loncar D. Projections of global mortality and burden of disease from 2002 to 2030. PLoS Med, 2006; 3:442.
Nakamura S, Kato K, Yoshida A, Fukuma N, Okumura Y, Ito H, Mizuno K. Prognostic value of depression, anxiety, and anger in hospitalized cardiovascular disease patients for predicting adverse cardiac outcomes. Am J Cardiol, 2013; 111:1432-1436.

O'Brien P, Oyebode F. Psychotropic medication and the heart. Advances in Psychiatric Treatment, 2003; 9:414-423.

Ormel J, Von Korff M, Burger H, Scott K, Demyttenaere K, Huang HQ, Posada-Villa J, Pierre Lepine J, Angermeyer MC, Levinson D, de Girolamo G, Kawakami N, Karam E, Medina-Mora ME, Gureje O, Williams D, Haro JM, Bromet EJ, Alonso J, Kessler R. Mental disorders among persons with heart disease - results from World Mental Health surveys. Gen Hosp Psychiatry, 2007; 29:325-334.

Player MS, Peterson LE. Anxiety disorders, hypertension, and cardiovascular risk: a review. Int J Psychiatry Med, 2011; 41:365-377.

Roest AM, Martens EJ, de Jonge P, Denollet J. Anxiety and risk of incident coronary heart disease: a meta-analysis. J Am Coll Cardiol, $2010 ; 56: 38-46$

Serafini G, Pompili M, Innamorati M, Iacorossi G, Cuomo I, Della Vista M, Lester D, De Biase L, Girardi P, Tatarelli R. The impact of anxiety, depression, and suicidality on quality of life and functional status of patients with congestive heart failure and hypertension: an observational cross-sectional study. Prim Care Companion J Clin Psychiatry, 2010; 12.

Shell WE, Swan HJ. Treatment of silent myocardial ischemia with transdermal nitroglycerin added to beta-blockers and alprazolam. Cardiol Clin, 1986; 4:697-704.

Sola CL, Bostwick JM. Implantable cardioverter-defibrillators, induced anxiety, and quality of life. Mayo Clin Proc, 205; 80:232-237.

Strik JJ, Honig A, Lousberg R, van Os J, van den Berg EJ, van

Praag HM. Clinical correlates of depression following myocardial infarction. Int J Psychiatry Med, 2001; 31:255-264.

Thurston RC, Rewak M, Kubzansky LD. An anxious heart: anxiety and the onset of cardiovascular diseases. Prog Cardiovasc Dis, 2013; 55:524-537.

Uzzal M. 2014. Heart hospital clogged with overcrowding In Dhaka Tribune, Dhaka.

Vogel WH, Miller J, DeTurck KH, Routzahn BK. Effects of psychoactive drugs on plasma catecholamines during stress in rats Neuropharmacology, 1984; 23:1105-1108.

Wawruch M, Zikavska M, Wsolova L, Kuzelova M, Tisonova J, Gajdosik J, Urbanek K, Kristova V. Polypharmacy in elderly hospitalised patients in Slovakia. Pharm World Sci, 2008; 30:235-242.

WHO. 2011. Global status report on noncommunicable disaeses 2010, Geneva, World Health Organization.

WHO. 2012. ATC/DDD Index 2012. Vol. 2012, Oslo.

WHO. 2012. International Classification of Diseases (ICD).

Williams Jr RB. Do benzodiazepines have a role in the prevention or treatment of coronary heart disease and other major medical disorders? J Psychiatr Res, 1990; 24 Suppl 2:51-56.

Williams RB. Do benzodiazepines have a role in the prevention or treatment of coronary heart disease and other major medical disorders? J Psychiatr Res, 1990; 24 Suppl 2:51-56.

Windle A, Elliot E, Duszynski K, Moore V. Benzodiazepine prescribing in elderly Australian general practice patients. Aust N Z J Public Health, 2007; 31:379-381.

\section{How to cite this article:}

Nasir Uddin MM, Kabir MSH, Al-Amin MM, Hasan M, Emran TB, Islam MT, Islam MA, Ahmed S. Evaluation of prescription pattern of psycholeptic medications at hospital discharge for patients with cardiovascular diseases in national cardiovascular disease hospital of Bangladesh. J App Pharm Sci, 2017; 7 (03): 017-023. 